\title{
SEMANTIC MANAGEMENT: APPLICATION OF ONTOLOGIES FOR THE INTEGRATION OF MANAGEMENT INFORMATION MODELS
}

\author{
Jorge E. López de Vergara, Víctor A. Villagrá, Julio Berrocal, Juan I. Asensio ${ }^{\dagger}$, and \\ Roney Pignaton \\ Dpto. Ingeniería de Sistemas Telemáticos, Universidad Politécnica de Madrid, Spain. \\ \{jlopez, villagra, berrocal, jasensio,roney\}@dit.upm.es \\ ${ }^{\dagger}$ Visiting researcher from Universidad de Valladolid, Spain
}

\begin{abstract}
The multiplicity of Network Management models (SNMP, CMIP, DMI, WBEM...) has raised the need of defining multiple mechanisms to allow the interoperability among all involved management domains. One basic component of such interoperability is the mapping between the information models that each domain specifies. These mappings, usually carried out with syntactical translations, can reach the semantic level by using ontologies. This article shows the advantages of using formal ontology techniques to improve the integration of current network management models.
\end{abstract}

Keywords: Ontology, Management Information Models Integration, Semantic Mapping, Behavior Information.

\section{INTRODUCTION}

Different standardization efforts for a unique integrated management model have set up several models: different management domains currently exist using IETF's SNMP, ISO's CMIP, DMTF's DMI or even OMG's CORBA. The definition of interoperability mechanisms among these models has become essential to perform an integrated management in a multiple domain scenario. Existing studies about this topic [1] divide the problem in issues related to their diverse communication protocols and information models: If a rule set can be specified that translates both the access and the definition of the information, interoperability is possible.

DMTF's WBEM management model includes some features to deal with this heterogeneity. In this new model, access interoperation is solved by using providers that act as gateways, but information interoperability is still an obstacle: CIM,

The original version of this chapter was revised: The copyright line was incorrect. This has been corrected. The Erratum to this chapter is available at DOI: 10.1007/978-0-387-35674-7_66 
WBEM's Common Information Model, defines three different mappings, which are technique, recast and domain [2]. However, the fact is that usual translations are at most recast mappings. Recasts only give a syntactic-equivalent definition that is not integrated in the semantic hierarchy of the CIM schemas, which could only be done with domain mappings.

Domain mappings are not easy to define, because they cannot be done automatically. To solve this problem, the knowledge representation discipline known as Ontology can be useful, because it provides all necessary constructs to add semantics to described information. In fact, some ontology tools exist that assist in the information merging and mapping task [3].

The following section introduces ontologies and compares them to CIM. Next, a method is proposed that applies ontology techniques to the integration of different management information specifications. Finally, some conclusions are presented.

\section{ONTOLOGIES}

An ontology can be defined as "an explicit and formal specification of a shared conceptualization" [4]. Briefly, it can be said that an ontology is the definition of a set of concepts, its taxonomy, interrelation and the rules that govern such concepts. In this way, existing management information models could be understood as a kind of ontologies: Models like CIM define the information of the management domain in a formal way and by common consent of working groups. However, they do not incorporate axioms or constraints that provide the additional semantics usually included in the so called heavyweight ontologies, and that would eventually enable the inference of knowledge based on existing one.

Ontologies are usually defined following a pyramidal structure in which more general and also more reusable ontologies are at the bottom level, and more usable and also more specific and less reusable are at the top [5]. CIM has a similar structure although it lacks both a General Common Ontology and any Task Ontology levels (see figure 1).

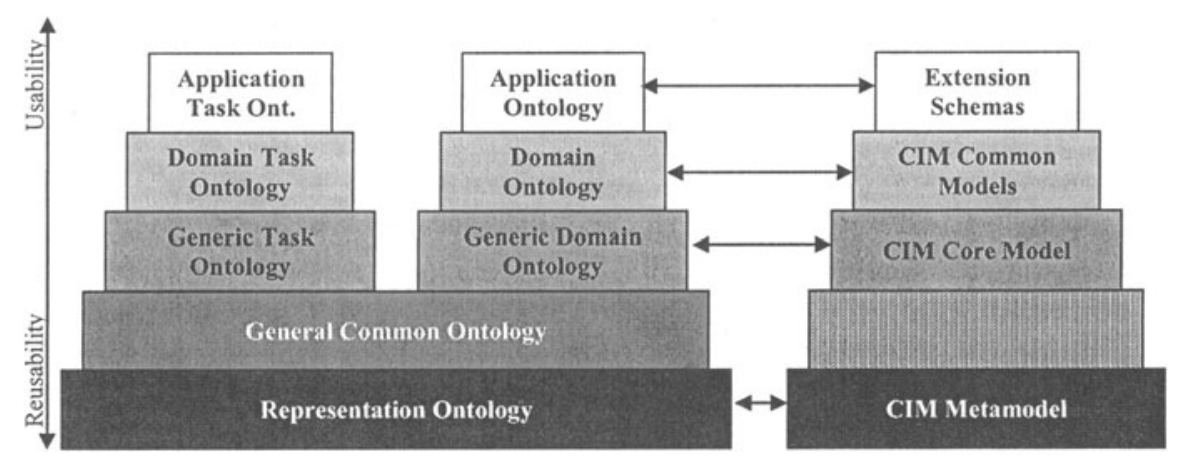

Figure 1. Correspondence between CIM and Ontology architectures. 


\section{APPLYING ONTOLOGIES TO NETWORK MANAGEMENT INTEGRATION}

As stated before, semantic interoperability is not completely achieved in CIM and thus, it should be extended. This section proposes a method to create a network management information model based on formal ontologies. The resulting ontology would be an extension of CIM, adding the necessary axioms and constraints to obtain a heavyweight ontology. Furthermore, this CIM-based ontology should also contain all information defined in other management models. This can be achieved by merging every model with CIM, including all necessary mapping rules.

Therefore, a set of steps can be defined to obtain the desired management global ontology, which can be used by a manager as an interoperable information model:

1. Translate all management information models to work with a single ontology representation language.

2. Merge the models in a global ontology, defining at the same time mapping rules between the global ontology and each model.

3. Add a set of formulae or axioms to the ontology to make it heavyweight.

The merging and mapping tasks can be assisted by means of ontology tools. In this case, a merging of CIM and SNMP MIBs has been done with one of such tools [6], using a subset of CIM and the whole HOST-RESOURCES-MIB (see figure 2). Both MOF and SMI specifications were manually translated to be readable by the ontology tool.

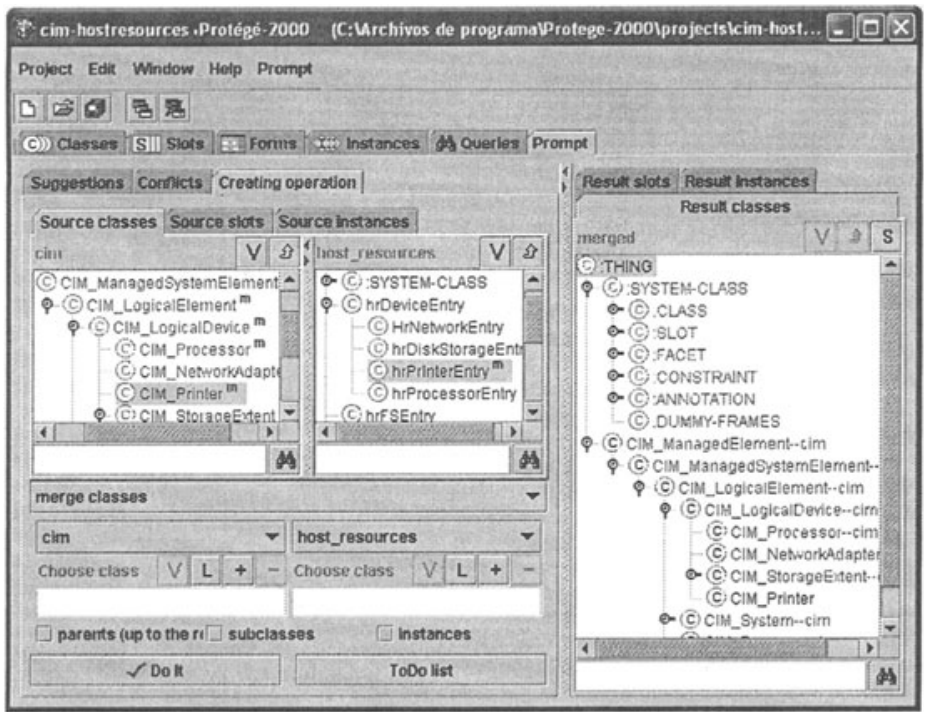

Figure 2. Merging CIM and HostResources with an ontology tool

With this approach, a manager can use the merged ontology to have a unified view of all the information it manages and translate it, by applying the mapping 
rules, into each domain information model taking into account the semantics of the concepts.

Constraints contained in the description of an OBJECT-TYPE or a CIM Property are defined in natural language and cannot be automatically enforced by a manager. If a formal definition of these constraints is provided, specified management information can be used to define certain manager behavior rules. Ontology tools also allow the definition of such axioms or constraints to complete the management ontology: For instance, the following rule could be set to impose that "the AvailableSpace of a CIM_FileSystem instance is going to be greater than a $10 \%$ of the FileSystemSize":

( defrange ?fs :FRAME CIM_FileSystem )

( forall ?fs ( > (AvailableSpace ?fs $)(* 0.10($ FileSystemSize ?fs $)))$

\section{CONCLUSIONS AND FUTURE WORK}

This paper has presented a novel approach in which the definition of an ontology-based management information model has been proposed. The advantages of this approach can be applied when trying to map and merge different management models from a semantic viewpoint. Also, ontology axioms and constraints provide a way to define the behavior related to the information model.

Current developments include a program that translates MOF and SMI specifications automatically to RDFS and DAML+OIL, dealing also with SMI particularities. At the same time, the ontology tool [6] is being adapted to the peculiarities of the management information when merging and mapping it.

\section{REFERENCES}

[1] Pramod Kalyanasundaram, Adarshpal S. Sethi, "Interoperability Issues in Heterogeneous Network Management", in Journal of Network and Systems Management, Vol. 2, No. 2, June 1994.

[2] Distributed Management Task Force, Inc., Common Information Model Specification version 2.2, DMTF Document DSP004, June 1999.

[3] Jorge E. López de Vergara, Víctor A. Villagrá, Julio Berrocal, "Semantic Management: advantages of using an ontology-based management information meta-model", in Proceedings of the HP Openview University Association Ninth Plenary Workshop (HPOVUA'2002), Böblingen, Germany, June 2002.

[4] R. Studer, V.R. Benjamins, D. Fensel, "Knowledge Engineering: Principles and Methods", in Data \& Knowledge Engineering, Vol. 25, 1998.

[5] Asunción Gómez Pérez, V. Richard Benjamins, "Overview of Knowledge Sharing and Reuse Components: Ontologies and Problem-Solving Methods", in Proceedings of the IJCAI-99 Workshop on Ontologies and Problem-Solving Methods (KRR5), Stockholm, Sweden, August 1999.

[6] Natalya F. Noy, "Managing Multiple Ontologies in Protégé-2000", in Proceedings of the Fifth International Protégé-2000 Workshop, Newcastle, England, July 2001. 